\title{
Ultrasonographic Evaluation of Needle Insertion Site for the Flexor Pollicis Longus
}

\author{
Seung Min Lee, MD, Kihoon Kim, MD, Sang Min Lee, MD, Hyun Seok Lee, MD \\ Department of Rehabilitation Medicine, VHS Medical Center, Seoul, Korea
}

\begin{abstract}
Objective To establish the safest approach to needle electrode insertion into the flexor pollicis longus (FPL) regarding possible needle injury to the superficial radial nerve (SRN) or radial artery by ultrasonography. Methods We evaluated 54 forearms of 27 healthy subjects. Three levels were defined in the forearm. Level 1 is the junction of the middle and distal third of the forearm, level 3 is the midpoint of forearm length, and level 2 is the midpoint between two levels. At each level, the distance between the most prominent point of the radius and the SRN (region A), the distance between the SRN and the radial artery (region B), and the depth from the skin surface to the FPL were measured.

Results The distance of region A was $1.20 \pm 0.41 \mathrm{~cm}$ in level $1,1.62 \pm 0.45 \mathrm{~cm}$ in level 2 , and $1.95 \pm 0.49 \mathrm{~cm}$ in level 3. The distance of region B was $1.02 \pm 0.29 \mathrm{~cm}$ in level $1,0.61 \pm 0.24 \mathrm{~cm}$ in level 2 , and $0.37 \pm 0.19 \mathrm{~cm}$ in level 3 . The depth from the skin surface to the FPL was $0.92 \pm 0.20 \mathrm{~cm}$ in level $1,1.14 \pm 0.26 \mathrm{~cm}$ in level 2, and $1.45 \pm 0.29 \mathrm{~cm}$ in level 3.

Conclusion The safest needle insertion point to the FPL is the middle of the forearm within approximately 0.8 $\mathrm{cm}$ from the most prominent point of the radius. We recommend that the needle is inserted at the above point perpendicular to the skin surface until the needle meets the FPL at a depth of approximately $1.45 \mathrm{~cm}$ from the skin surface.
\end{abstract}

Keywords Electromyography, Ultrasonography, Forearm, Needlestick

\section{INTRODUCTION}

The flexor pollicis longus originates from the anterior surface of the body of the radius, the adjacent part of the interosseous membrane and inserts into the base of the

Received July 2, 2012; Accepted October 4, 2012

Corresponding author: Kihoon Kim

Department of Rehabilitation Medicine, VHS Medical Center, 53 Jinhwangdo-ro 61-gil, Gangdong-gu, Seoul 134-791, Korea

Tel: +82-2-2225-1166, Fax: +82-2-2225-1579, E-mail: kkh702@korea.ac.kr

(c) This is an open-access article distributed under the terms of the Creative Commons Attribution Non-Commercial License (http://creativecommons. org/licenses/by-nc/3.0) which permits unrestricted noncommercial use, distribution, and reproduction in any medium, provided the original work is properly cited.

Copyright (c) 2013 by Korean Academy of Rehabilitation Medicine distal phalanx of the thumb. The flexor pollicis longus is the flexor of the distal phalanx of the thumb and may assist in flexing the wrist when the thumb is fixed. Since this muscle receives innervations from the anterior interosseous nerve, a branch of the median nerve, electrodiagnostic evaluation of this muscle is useful in patients with difficulty gripping objects for diagnosing anterior interosseous neuropathy and identifying the site and determining the severity of the lesion $[1,2]$. In addition, the flexor pollicis longus should be the target in controlling spasticity by chemodenervation since it may cause thumb curling related to motor neuron disease [3].

A careful needle electrode approach is needed because the superficial radial nerve and radial artery are 
located near the flexor pollicis longus muscle. There are two methods used to place the needle electrode. In one of the methods, the needle is inserted at the junction of the middle and distal third of the forearm between the brachioradialis and flexor carpi radialis [4]. In the other method, the needle electrode is inserted in the middle of the forearm, from the radial aspect just volar to the radius [5].

Electrodiagnostic procedures are rarely thought to be associated with any significant side effect. But, needle electrode examination is an invasive procedure and it has the potential to be associated with iatrogenic complications, including bleeding, infection, nerve injury, pneumothorax, and other local trauma. Thus, local nerve trauma could result from a direct intraneural puncture from an electromyography needle [6].

Little is known about the method for placing the needle to the flexor pollicis longus muscle without causing damage to a neurovascular bundle. Furthermore, the ultrasonographic evaluation of the needle electrode insertion site into the flexor pollicis longus has not yet been studied.

Therefore, the purpose of this study was to establish the safest approach to needle electrode insertion, and to standardize a needle electrode insertion method.

\section{MATERIALS AND METHODS}

\section{Subject}

We recruited 27 healthy subjects (15 males and $12 \mathrm{fe}$ males; mean age \pm standard deviation [SD], 27.8 \pm 6.44 years), who had no history of neuromuscular disease, exhibited normative findings in a neurologic examination, and were currently not taking any medication. Measurements were taken on both forearms for all subjects, thus, there were 54 forearms examined in total. This study protocol was approved by the Institutional Review Board. Every subject participated voluntarily in this study and consent forms were signed by each of them.

Height, length and circumference of both forearms were measured. The length of the forearm was defined as the distance from the antecubital skin crease to the distal wrist crease with the forearm supinated and the elbow at $180^{\circ}$ in a sitting position. The forearm circumference was measured at the mid-forearm level.

\section{Ultrasonographic examination}

A high-resolution, real time ultrasonography was performed by an expert physiatrist using the ACCUVIX XQ system (Medison, Seoul, Korea) interfaced with a 5 to $12 \mathrm{MHz}$ linear array transducer. The ultrasonographic images were converted to a Digital Imaging and Communication in Medicine (DICOM) file and saved in picture archiving and communication system mView 5.3 (Marotech, Seoul, Korea). Then, with measuring tools we determined the parameters.

Ultrasonographic examinations of the flexor pollicis longus were performed in transverse view with subjects in the sitting position with their forearms supinated. For preventing the compression effect of transducer, water was used as a medium instead of gel.

Three levels to be scanned were marked in the forearm. Level 1 is the junction of the middle and distal third of the forearm, level 3 is the middle point of forearm length, and level 2 is the midpoint of level 1 and level 3 (Fig. 1). At each level, an ultrasonographic image of the most prominent point of the radius (landmark a), midpoint of the superficial radial nerve (landmark b) and midpoint of the radial artery (landmark c) was obtained. The most prominent point of radius was defined as the closest bone point to the skin surface in the ultrasonographic image. In addition, we obtained three points (Sa, $\mathrm{Sb}, \mathrm{Sc}$ ) on the skin surface where a line makes distance a

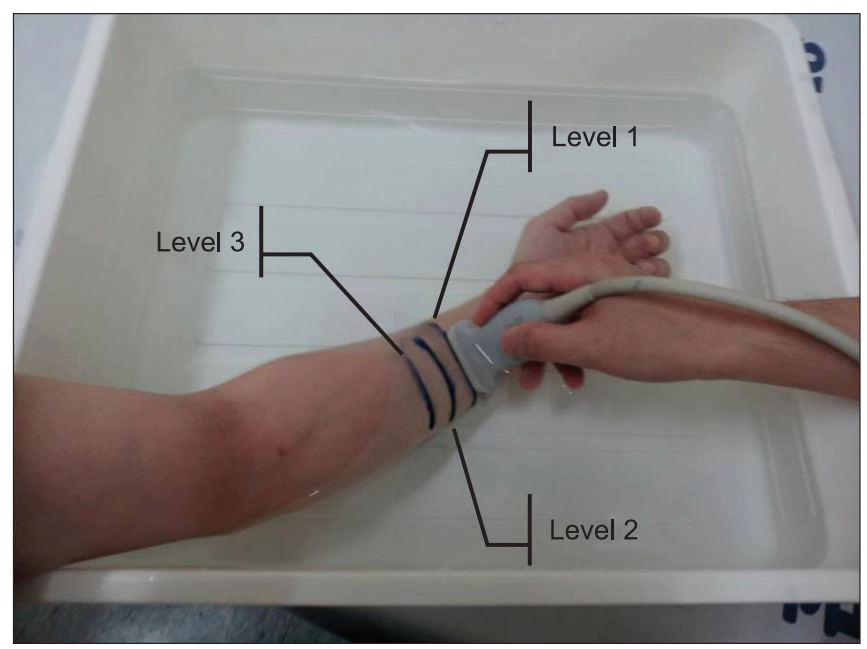

Fig. 1. Ultrasonography of the forearm in water bath with the transducer placed on the three levels of the forearm in transverse plane. Level 1 , the junction of middle and distal third of the forearm; level 2, the midpoint of level 1 and level 3; level 3, the midpoint of the forearm. 

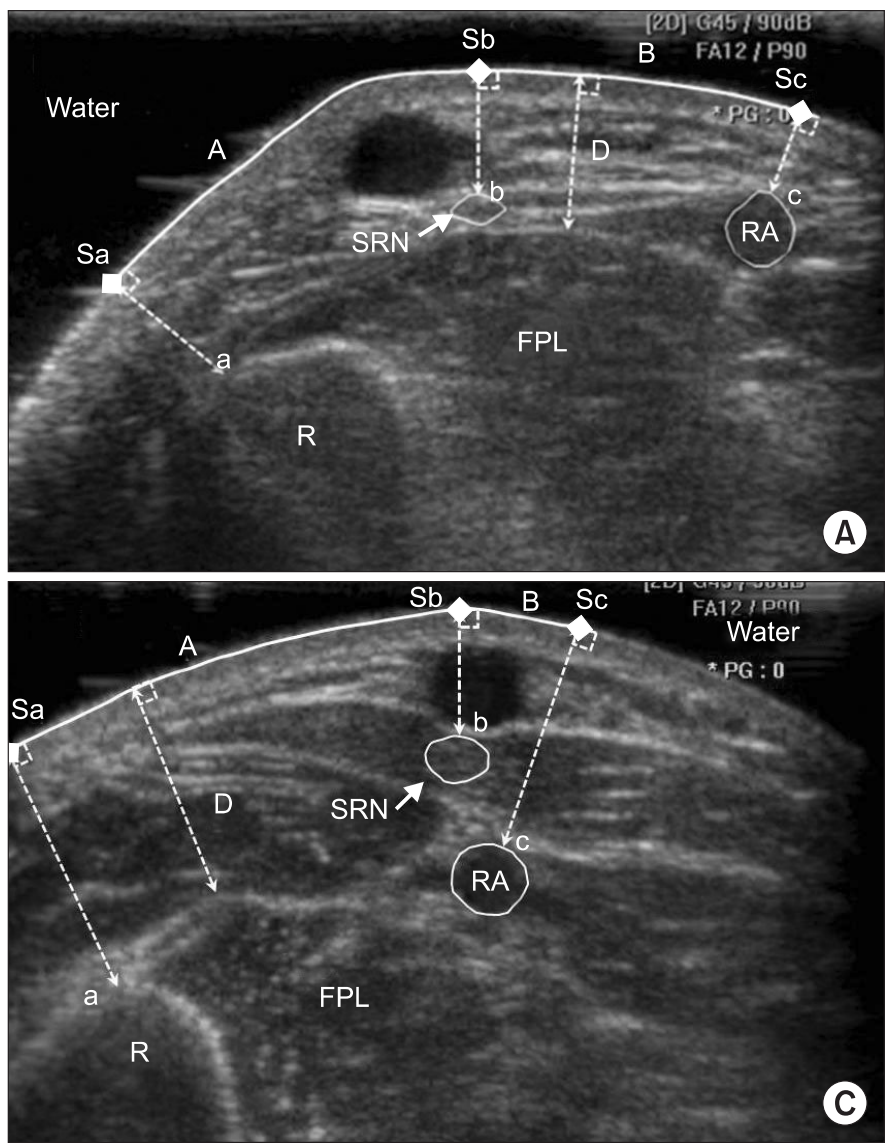

minimum from above each landmark to the skin surface because the line will be perpendicular to the skin surface when the distance is minimum. Then, we measured the distance between Sa and Sb (region A), and the distance between $\mathrm{Sb}$ and $\mathrm{Sc}$ (region $\mathrm{B}$ ) along the skin surface line using a distance measuring tool (Fig. 2). The depths to the flexor pollicis longus (distance D), the horizontal diameters of superficial radial nerves and radial arteries were measured at each level, too. Among six regions (region $\mathrm{A}$ and $\mathrm{B}$ at each of the three levels), the region with the greatest distance was determined as the safest needle insertion site.

\section{Statistical analysis}

The Student $\mathrm{t}$-test was performed to compare the forearm length, circumference, distance of region A, distance of region $\mathrm{B}$, and depth to the flexor pollicis longus between men and women. The distances, depth, and width of nerve and artery in levels 1,2 , and 3 were compared using an analysis of variance for repeated measurements (RM-ANOVA) and Bonferroni correction. In order to test whether the sphericity assumption was met (i.e., vari-

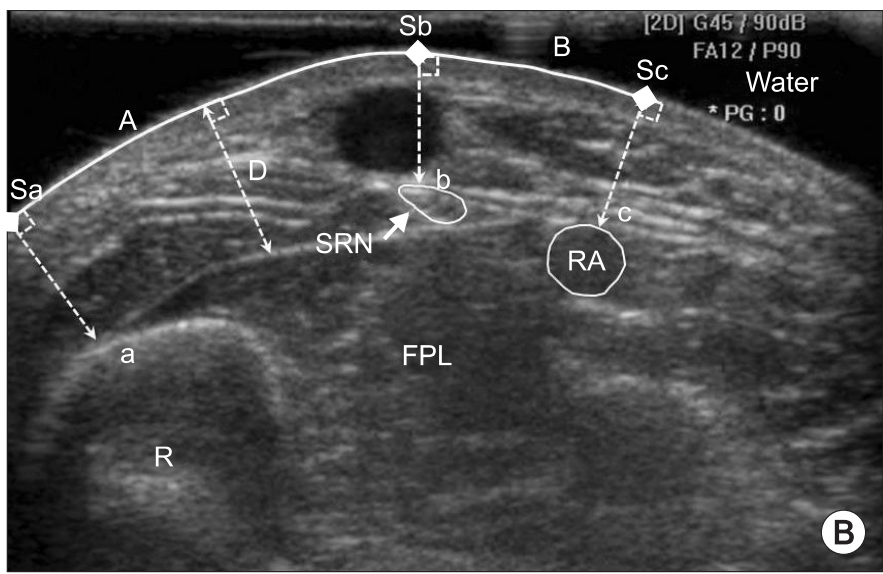

Fig. 2. Transverse view of ultrasonographic images of the forearm at the junction of middle and distal third of the foreram (level $1 ; \mathrm{A}$ ), at the midpoint between level 1 and level 3 (level 2; B), and at the midpoint of the forearm (level $3 ; \mathrm{C})$. a, most prominent point of the radius; $\mathrm{b}$, midpoint of the superficial radial nerve; $\mathrm{c}$, midpoint of the radial artery; Sa, skin surface point from a; Sb, skin surface point from b; Sc, skin surface point from c; A, distance between $\mathrm{Sa}$ and $\mathrm{Sb} ; \mathrm{B}$, distance between $\mathrm{Sb}$ and $\mathrm{Sc}$; $\mathrm{D}$, depth from the skin surface to the FPL; RA, radial artery; SRN, superficial radial nerve; $\mathrm{R}$, radius; FPL, flexor pollicis longus.

Table 1. Comparison of forearm length, circumference, distance of region $\mathrm{A}, \mathrm{B}$ and depth between men and women

\begin{tabular}{|c|c|c|}
\hline Parameter $(\mathrm{cm})$ & Male & Female \\
\hline Forearm length & $25.53 \pm 1.33$ & $23.52 \pm 0.94^{*}$ \\
\hline Forearm circumference & $21.76 \pm 1.41$ & $18.65 \pm 1.50^{*}$ \\
\hline \multicolumn{3}{|l|}{ Distance of region A } \\
\hline Level 1 & $1.26 \pm 0.42$ & $1.08 \pm 0.41$ \\
\hline Level 2 & $1.72 \pm 0.43$ & $1.46 \pm 0.46$ \\
\hline Level 3 & $2.07 \pm 0.49$ & $1.79 \pm 0.49$ \\
\hline \multicolumn{3}{|l|}{ Distance of region B } \\
\hline Level 1 & $1.08 \pm 0.27$ & $1.00 \pm 0.30$ \\
\hline Level 2 & $0.63 \pm 0.30$ & $0.59 \pm 0.16$ \\
\hline Level 3 & $0.37 \pm 0.22$ & $0.40 \pm 0.15$ \\
\hline \multicolumn{3}{|l|}{ Depth } \\
\hline Level 1 & $0.90 \pm 0.19$ & $0.95 \pm 0.20$ \\
\hline Level 2 & $1.12 \pm 0.26$ & $1.14 \pm 0.26$ \\
\hline Level 3 & $1.47 \pm 0.29$ & $1.38 \pm 0.29$ \\
\hline
\end{tabular}

Values are presented as mean \pm standard deviation.

${ }^{*} \mathrm{p}<0.05$. 
ance-covariance matrix is circular), we also performed a Mauchly's test of sphericity. If the sphericity assumption was violated $(\mathrm{p}<0.05)$, the Huynh-Feldt correction was used for estimating p-values. Also, if it was not violated, we could use exact $p$-values. Data were analyzed by the SPSS ver. 18.0 (SPSS Inc., Chicago, IL, USA) and statistical significance was accepted for $\mathrm{p}$-values less than 0.05 .

\section{RESULTS}

The mean \pm SD of height, length of the forearm, and circumference of the forearm were $170.52 \pm 7.26,24.74 \pm 1.50$, $20.50 \pm 2.02 \mathrm{~cm}$, respectively. There was no significant difference between results obtained from men and women except forearm length and circumference (Table 1).

The distance of region A was $1.20 \pm 0.41 \mathrm{~cm}$ (range, 0.50 to $2.37 \mathrm{~cm}$ ) in level $1,1.62 \pm 0.45 \mathrm{~cm}$ (range, 0.73 to 2.80 $\mathrm{cm}$ ) in level 2, and $1.95 \pm 0.49 \mathrm{~cm}$ (range, 0.88 to $2.99 \mathrm{~cm}$ ) in level 3 and the sphericity assumption was violated $(\mathrm{p}=0.02)$. The distance of region $\mathrm{B}$ was $1.02 \pm 0.29 \mathrm{~cm}$ (range, 0.43 to $1.57 \mathrm{~cm}$ ) in level $1,0.61 \pm 0.24 \mathrm{~cm}$ (range, -0.31 to $1.22 \mathrm{~cm}$ ) in level 2 , and $0.37 \pm 0.19 \mathrm{~cm}$ (range, -0.25 to $0.67 \mathrm{~cm}$ ) in level 3 and the assumption was not violated $(\mathrm{p}=0.07)$. Region A in level 3 demonstrated the greatest distance among the six regions compared. The depth to the flexor pollicis longus was significantly greatest at level $3(0.92 \pm 0.20 \mathrm{~cm}$ in level $1,1.14 \pm 0.26 \mathrm{~cm}$ in level 2 , and $1.45 \pm 0.29 \mathrm{~cm}$ in level 3 ), and the assumption was violated $(\mathrm{p}=0.01)$. The horizontal diameter of the superficial radial nerve was not significantly different $(0.24 \pm 0.04 \mathrm{~cm}$ in level $1,0.24 \pm 0.04 \mathrm{~cm}$ in level 2 , and $0.24 \pm 0.04 \mathrm{~cm}$ in level $3)$ and that of the radial artery was not significantly different, either $(0.27 \pm 0.04 \mathrm{~cm}$ in level $1,0.27 \pm 0.03 \mathrm{~cm}$ in level 2 , and $0.27 \pm 0.03 \mathrm{~cm}$ in level 3) (Table 2).

\section{DISCUSSION}

The identification of an optimal needle electrode insertion point is important for obtaining optimal motor unit action potential and minimizing patient discomfort and

Table 2. Comparison of ultrasonographic parameters among the forearm levels with transverse view (n=54)

\begin{tabular}{lcc}
\multicolumn{1}{c}{ Parameter } & Mean \pm SD $(\mathbf{c m})$ & Range $(\mathbf{c m})$ \\
\hline Level 1: junction of middle and distal third of the forearm & & \\
\hline Distance of region A & $1.20 \pm 0.41^{\mathrm{a})}$ & $0.50-2.37$ \\
\hline Distance of region B & $1.02 \pm 0.29^{\mathrm{b})}$ & $0.43-1.57$ \\
\hline Depth & $0.92 \pm 0.20^{\mathrm{c})}$ & $0.59-1.45$ \\
\hline Nerve diameter (horizontal) & $0.24 \pm 0.04$ & $0.18-0.44$ \\
\hline Artery diameter (horizontal) & $0.27 \pm 0.04$ & $0.22-0.39$ \\
\hline Level 2: midpoint between the level 1 and level 3 & & \\
Distance of region A & $1.62 \pm 0.45^{\mathrm{a})}$ & $0.73-2.80$ \\
\hline Distance of region B & $0.61 \pm 0.24^{\mathrm{b})}$ & $-0.31-1.22$ \\
\hline Depth & $1.14 \pm 0.26^{\mathrm{c}}$ & $0.77-1.71$ \\
\hline Nerve diameter (horizontal) & $0.24 \pm 0.04$ & $0.17-0.41$ \\
\hline Artery diameter (horizontal) & $0.27 \pm 0.03$ & $0.22-0.40$ \\
Level 3: midpoint of the forearm & & \\
Distance of region A & $1.95 \pm 0.49^{\mathrm{a})}$ & $0.88-2.99$ \\
\hline Distance of region B & $0.37 \pm 0.19^{\mathrm{b})}$ & $-0.25-0.67$ \\
\hline Depth & $1.45 \pm 0.29^{\mathrm{c})}$ & $0.83-2.08$ \\
\hline Nerve diameter (horizontal) & $0.24 \pm 0.04$ & $0.17-0.39$ \\
\hline Artery diameter (horizontal) & $0.27 \pm 0.03$ & $0.22-0.40$ \\
\hline
\end{tabular}

Distance of region A, skin surface distance from the most prominent point of radius to midpoint of superficial radial nerve; Distance of region B, skin surface distance from midpoint of superficial radial nerve to midpoint of radial artery; SD, standard deviation.

a) $\mathrm{p}<0.05$, among three forearm levels (distance of region A). ${ }^{\mathrm{b})} \mathrm{p}<0.05$, among three forearm levels (distance of region $\mathrm{B}$ ). ${ }^{c} \mathrm{p}<0.05$, among three forearm levels (depth). 
complications. There were several studies and articles for evaluating needle electrode insertion sites into the tibialis posterior [7-10]. However, there was no study regarding an insertion site for the flexor pollicis longus. To our knowledge, this is the first study to establish the safest approach to needle electrode insertion into the flexor pollicis longus.

Ultrasonographic imaging enables excellent direct and real-time inspection. It also has the advantages of easy accessibility, cost effectiveness. Ultrasonography facilitates direct visualization during needle placement and enhances the safety and accuracy of electromyography [11].

Electromyography has been used to assist in chemodenervation [3]. But, in patients with significant paralysis or intelligence so low they are unable to follow commands, electromyography-guided chemodenervation is difficult to be applied to them. In that case, there needs to be an alternative way. In previous study, the ultrasonographyguided injection technique confirms correct placement of needle and injection of the drug and it can be minimize complications $[12,13]$. Based on these studies, ultrasonographic evaluation of the needle electrode insertion site can be standardize needle electrode insertion method and minimize complications of the needle electrode insertion.

We measured forearm length from the antecubital skin crease to the distal wrist crease. But, in other studies, forearm length was measured from the ulnar styloid process to olecranon $[14,15]$. This method is more accurate than our method. Otherwise, our measurement method is easier than the other method because of the supinated forearm position while inserting a needle to the flexor pollicis longus. Hence, Lee [4] and Perotto [5] measured the forearm length in the same way as us.

We used the water bath evaluation technique. The linear probe has difficulty conforming to the curvature of the forearm without compression. If the compression is applied to skin to contact the probe, the internal structures may be moved and soft tissue can be compressed leading to measurement error. However, with the use of a water bath, any curvature can be achieved without compression by increasing the distance from the probe to the skin [16]. We can prevent abrupt changes in contour, and obtain better resolution of near skin surface structures.

Region B in level 2 and level 3 represented compara- tively small distance and even had negative values which mean the radial artery rather than the superficial radial nerve was closer to the radius. Needle insertion into this area may exhibit a relatively high risk of damage to the nerve or artery than region A. In the level 3, the minimum distance of region A was $0.88 \mathrm{~cm}$ and that of region B was $-0.25 \mathrm{~cm}$. But, each of the values is from a different subject and the minimum distance from the most prominent point of the radius to neurovascular bundle was 0.88 $\mathrm{cm}$ in level 3.

The safest needle insertion site into the flexor pollicis longus in our study was between the most prominent point of the radius and the superficial radial nerve (region A) at the midpoint of the forearm (level 3) because region A in level 3 revealed the greatest distance compared to the other regions. Although the mean depth to the flexor pollicis longus at level 3 was deeper than that at the other levels, the deepest point was $2.08 \mathrm{~cm}$ in our study and the point could be reached via a $5-\mathrm{cm}$ needle. Therefore, the depth is not a considerable factor for needle insertion into the flexor pollicis longus.

To insert the needle electrode safely, according to the result of our study, we recommend drawing an imaginary midpoint line between the antecubital crease and the wrist distal crease. Then, insert the needle within approximately $0.8 \mathrm{~cm}$ from the most prominent point of the radius perpendicularly. Although palpation of the patient's radial arterial pulse is helpful, it is difficult to feel the pulse due to the muscle bulk of the forearm. To reach the flexor pollicis longus, the needle should pass through the extensor carpi radialis. In our study, the depth varies from patient to patient (range, 0.83 to $2.08 \mathrm{~cm}$ ), but the average was approximately $1.45 \mathrm{~cm}$.

\section{Study limitation}

The main limitation of our study is the quantity of subjects. Also, there is no confirmation by ultrasonography guided needle insertion. The summation of all of the electrical activity produced from muscle fibers make the motor unit action potential (MUAP), so MUAP morphology varies with the location of the needle electrode [17]. We evaluated the safest needle electrode insertion site, but we did not insert the needle to the flexor pollicis longus and confirm the MUAP morphology. Therefore, further studies are needed to increase the quantity of subjects and apply the actual needle electromyography. 
In conclusion, for needle insertion into the flexor pollicis longus, ultrasonography allows safer and more accurate approach. The results of this study demonstrate that the safest and most appropriate needle insertion point to the flexor pollicis longus is the middle of the forearm within about $0.8 \mathrm{~cm}$ from the most prominent point of the radius. We recommend that the needle is inserted at the above point perpendicular to the skin surface until the needle reaches the flexor pollicis longus at a depth about $1.45 \mathrm{~cm}$ from the skin surface.

\section{CONFLICT OF INTEREST}

No potential conflict of interest relevant to this article was reported.

\section{REFERENCES}

1. Jenkins DB. Hollinshead's functional anatomy of the limbs and back. 9th ed. Philadelphia: Saunders; 2009.

2. Seror P. Anterior interosseous nerve lesions: clinical and electrophysiological features. J Bone Joint Surg Br 1996;78:238-41.

3. Pathak MS, Nguyen HT, Graham HK, Moore AP. Management of spasticity in adults: practical application of botulinum toxin. Eur J Neurol 2006;13 Suppl 1:4250 .

4. Lee HJ, DeLisa JA. Manual of nerve conduction study and surface anatomy for needle electromyography. 4th ed. Philadelphia: Lippincott Williams \& Wilkins; 2004.

5. Perotto AO. Anatomical guide for the electromyographer: the limbs and trunk. 4th ed. Springfield: Charles C Thomas; 2005.

6. Al-Shekhlee A, Shapiro BE, Preston DC. Iatrogenic complications and risks of nerve conduction studies and needle electromyography. Muscle Nerve 2003;27:517-26.

7. Lee HJ, Bach JR, DeLisa JA. Needle electrode insertion into tibialis posterior: a new approach. Am J Phys Med Rehabil 1990;69:126-7.

8. Yang SN, Lee SH, Kwon HK. Needle electrode inser- tion into the tibialis posterior: a comparison of the anterior and posterior approaches. Arch Phys Med Rehabil 2008;89:1816-8.

9. Rha DW, Im SH, Lee SC, Kim SK. Needle insertion into the tibialis posterior: ultrasonographic evaluation of an anterior approach. Arch Phys Med Rehabil 2010;91:283-7.

10. Won SJ, Kim JY, Yoon JS, Kim SJ. Ultrasonographic evaluation of needle electromyography insertion into the tibialis posterior using a posterior approach. Arch Phys Med Rehabil 2011;92:1921-3.

11. Boon AJ, Alsharif KI, Harper CM, Smith J. Ultrasoundguided needle EMG of the diaphragm: technique description and case report. Muscle Nerve 2008;38:16236.

12. Willenborg MJ, Shilt JS, Smith BP, Estrada RL, Castle JA, Koman LA. Technique for iliopsoas ultrasoundguided active electromyography-directed botulinum a toxin injection in cerebral palsy. J Pediatr Orthop 2002;22:165-8.

13. Jordan SE, Ahn SS, Gelabert HA. Combining ultrasonography and electromyography for botulinum chemodenervation treatment of thoracic outlet syndrome: comparison with fluoroscopy and electromyography guidance. Pain Physician 2007;10:541-6.

14. Bechtold S, Rauch F, Noelle V, Donhauser S, Neu CM, Schoenau E, et al. Musculoskeletal analyses of the forearm in young women with Turner syndrome: a study using peripheral quantitative computed tomography. J Clin Endocrinol Metab 2001;86:5819-23.

15. Neu CM, Rauch F, Rittweger J, Manz F, Schoenau E. Influence of puberty on muscle development at the forearm. Am J Physiol Endocrinol Metab 2002;283: E103-7.

16. Blaivas M, Lyon M, Brannam L, Duggal S, Sierzenski P. Water bath evaluation technique for emergency ultrasound of painful superficial structures. Am J Emerg Med 2004;22:589-93.

17. Dumitru D, Amato AA, Zwarts M. Electrodiagnostic medicine. 2nd ed. Philadelphia: Hanley \& Belfus; 1995. 